\title{
Spatial monomer distribution for a flexible polymer in a good solvent
}

\author{
T. A. Witten, $J r^{a}$ \\ Physics Department, The University of Michigan, Ann Arbor, Michigan 48109
}

L. Schäfer ${ }^{\text {b) }}$

Institute for Theoretical Physics, University of Hannover, 3000 Hannover, Federal Republic of Germany (Received 22 August 1980; accepted 28 October 1980)

\begin{abstract}
We calculate the fourth moment $\left\langle r^{4}\right\rangle$ of the average monomer-monomer separation in a flexible linear polymer in the excluded volume limit. The ratio $\left\langle r^{4}\right\rangle /\left\langle r^{2}\right\rangle^{2}$ is a universal number expressible in terms of the exponents $\gamma$ and $\nu$. The ratio is $8 \%$ smaller for the excluded volume chain than for the ideal chain. To account for the excluded volume interaction we use field theory methods to renormalize the MayerFixman cluster expansion. Our approximation method of expanding in dimension of space minus four introduces an estimated uncertainty of $1 \%$. The end point moment ratio $\left\langle r_{c}^{4}\right\rangle\left\langle\left\langle r_{e}^{2}\right\rangle^{2}\right.$ behaves similarly.
\end{abstract}

\section{INTRODUCTION}

The spatial monomer distribution in a randomly coiling polymer is a fundamental property which can be measured directly by light ${ }^{1}$ or neutron ${ }^{2}$ scattering. These experiments measure the structure function $S(q)$, defined as the Fourier transform of the monomer density autocorrelation function $\langle\rho(0) \rho(r)\rangle-\langle\rho\rangle^{2}$. The structure function for a noninteracting chain of $n$ monomers, with no self-repulsion, has been understood since Debye's 1947 derivation $^{3}$ :

$$
S(q)=2 n^{2}\left(Q^{2}-1+e^{-Q^{2}}\right) / Q^{4},
$$

where $Q \sim q n^{1 / 2}$. However, the corresponding problem for a self-repelling chain remains unsolved. This is despite valuable formal ${ }^{4,5}$ and phenomenological ${ }^{6-10}$ contributions. By exploiting the renormalization symmetry of the polymer field theory ${ }^{11}$ one may establish a scaling law for $S(q)$ :

$$
S(q)=n^{2} f_{1}\left(B q n^{\nu}\right),
$$

where the critical exponent $\nu$ is a pure number which may be calculated with great precision. ${ }^{12}$ The quantity $B$ is a system-dependent constant and the "scaling function" $f_{1}(x)$ has the form $1-x^{2}+O\left(x^{4}\right)$ for small $x$. This scaling law and the predicted $\nu$ agree well with experiments, ${ }^{13}$ provided the polymer is sufficiently long and the solvent sufficiently good.

We here calculate $f_{1}(x)$ approximately for small $x$ using methods of renormalized field theory. Specifically, we calculate the coefficient of $x^{4}$, which we denote by $\zeta$, to first order in $\epsilon \equiv 4-d$, where $d$ denotes the dimension of space. This $\zeta$ varies as the fourth moment of the monomer-monomer distance: $\zeta=3 / 10\left\langle r^{4}\right\rangle\left\langle r^{2}\right\rangle^{-2}$ in three di mensions. For an ideal chain $\zeta=3 / 4$. For the excluded volume limit we find $\zeta=0.690 \pm 1 \%-8 \%$ smaller. This suggests that the density profile drops off more sharply at large distance than in an ideal chain. Our result yields a precise estimate in three dimensions and it involves the exponent $\nu$. Our result also depends on a

\footnotetext{
a) Supported by NSF polymer program under DMR77 14853.

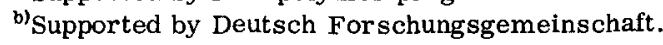

second exponent $\gamma$ which has not thus far been measured in polymers. This $\gamma$ is the exponent governing the $n$ dependence of the polymer partition function. ${ }^{7}$

The appealing "swollen Gaussian" argument of Ptitsyn" and Benoit ${ }^{10}$ allows one to estimate $\zeta$. Here one assumes that any two monomers $i$ and $i+k$ are distributed in space as in an ideal chain, except that their mean separation goes as $k^{\nu}$ instead of $k^{1 / 2}$. Using the Flory value $\nu=3 / 5$, one finds that $\zeta$ is $10 \%$ larger than in an ideal chain, in contrast to our result.

Our method is equivalent to the field theory of polymers formulated by De Gennes ${ }^{14}$ and Des Cloizeaux ${ }^{15}$ and extended by ourselves. ${ }^{11}$ Thus, our theory may be viewed as a Landau-Ginzberg-Wilson ${ }^{18}$ theory for a phase transition of a system with a zero-component or der parameter. ${ }^{14}$ On the other.hand, our theory may be viewed as an extension of Fixman's ${ }^{17}$ adaptation of the Mayer cluster expansion to polymers. In this article we use the Fixman language to describe the renormalization process, thus avoiding features of the field theory not needed here. We may thus motivate as simply as possible the use of the epsilon expansion method for polymers. In Sec. II we recall the cluster expansion prescription. In Sec. III we show how the expansion is renormalized to treat the excluded volume limit. In Sec. IV we calculate $\zeta$ and discuss our results.

\section{CLUSTER EXPANSION}

Our system is a grand canonical ensemble of single chains including all lengths $n$, at temperature $T$, with a monomer potential $k T s$ controlling the average length. A chain may be represented ${ }^{18}$ as a sequence of points $r_{i}$ on a (hyper)cubic lattice in $d$ dimensional space. Each $r_{i}$ is one lattice spacing $l$ from its predecessor. A short-ranged repulsive potential $v(r)$ acts between every pair of monomers at distance $r$. We may calculate the structure function $S(q)$ in terms of a certain constrained partition function $Y(y, s)$ defined by

$Y(y, s)=\sum_{n=0}^{\infty} \sum_{(0, y)}^{\prime} e^{-s n} \exp \left[-\sum_{i<j} v\left(r_{i}-r_{j}\right) / k T\right]$. 


\section{$+a$}
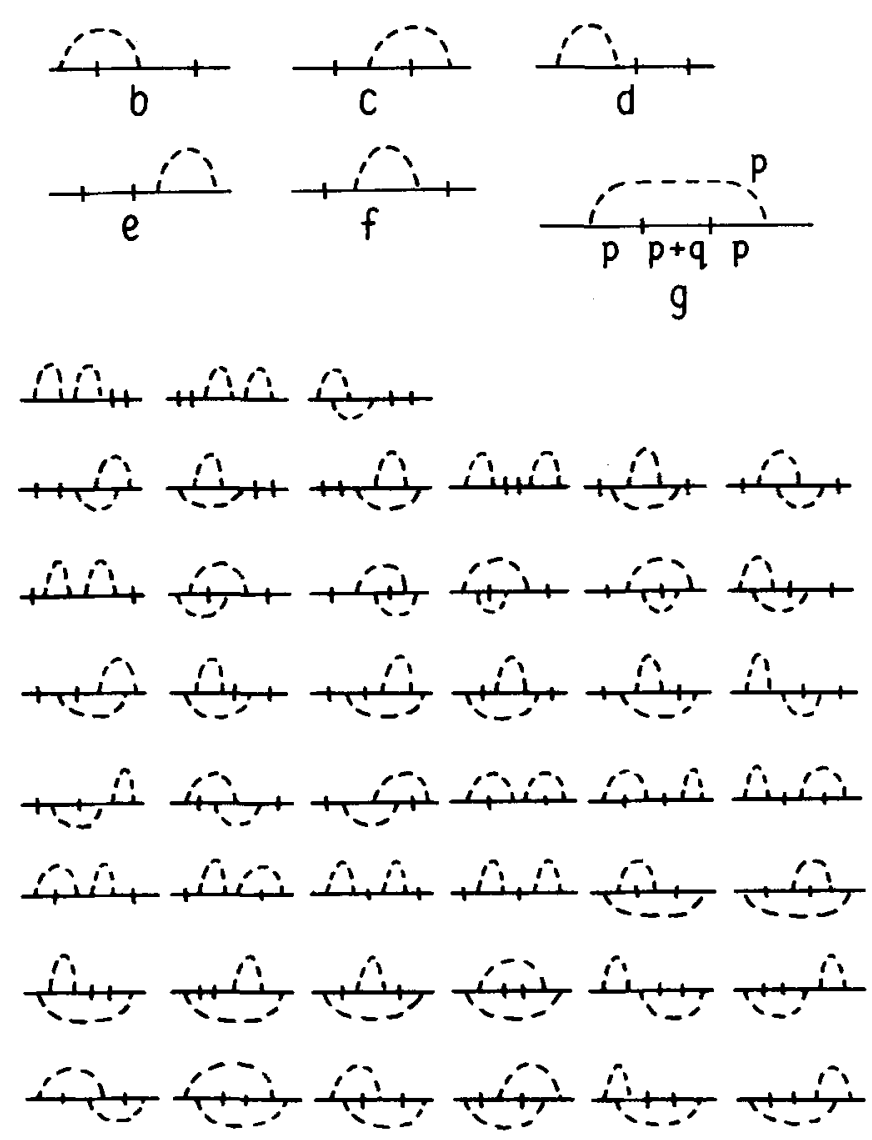

FIG. 1. The single zeroth order 4 ! $/\left(2^{2} 1\right.$ !) first-order and $6 ! /\left(2^{3} 2 !\right)$ second-order diagrams for $\tilde{Y}(q, t)$ defined in the text. Letters (a) $-(\mathrm{g})$ label the zeroth- and first-order diagrams. In diagram $(\mathrm{g})$ the wave vector variables for each $\tilde{Q}_{0}$ and $\tilde{f}$ are indicated.

The primed sum is a sum over allowed configurations of the chain without the repulsion, but constrained so that some point of the chain passes through the origin and some other point passes through the point $y$. Apart from a $q$-independent factor the grand canonical structure function $S(q, s)$ is the spatial Fourier transform of $Y(y$, $s)$. We denote this transform by $\tilde{Y}(q, s)$. Given $\tilde{Y}(q$, $s$ ) one may readily determine the monodisperse structure function $S_{n}(q)$, as we show below. In calculating $\tilde{Y}(q, s)$ it is useful to define the fixed-end partition function $Q(y$, $s)$; this is the weighted number of configurations which begin at the origin and end at $y$ :

$Q(y, s)=\sum_{n=0}^{\infty} \sum_{\left\{r_{0}=0, r_{1}, \cdots, r_{n}=y\right\}} e^{-s n} \exp \left[-\sum_{i<j} v\left(r_{i}-r_{j}\right) / k T\right]$.

The Fourier transform of $Q(y, s)$ is denoted $\tilde{Q}(q, s)$; the $\bar{Q}(q, s)$ for an ideal chain, denoted $\tilde{Q}_{0}(q, s)$, has a simple closed form in our lattice model. To obtain the long chain behavior we shall be interested in that range of $s$ for which the average length $N$ is much greater than 1. This occurs near a critical value $s_{c}$, at which $N \rightarrow \infty$. For $s<s_{c}, \tilde{Y}$ and $\tilde{Q}$ are undefined. In the lattice model with no repulsion $s_{c}=\ln (2 d)$, and with repulsion $s_{c}$ decreases.

The calculation of $\tilde{Y}$ is based on a cluster expansion ${ }^{17,19}$ in powers of $f(r) \equiv\left(e^{-v(r) / k T}-1\right)$. The exponential inside Eq. (3), and thence $\bar{Y}$ itself, may be expressed as a power series in $f(r)$. The cluster diagrams for the expansion (Fig. 1) represent the polymer chain with the constrained points, and show the (negative) Mayer factors $f$ acting between various parts of the chain. These diagrams are simpler to calculate than those of the original Fixman formulation, owing to our use of the monomer chemical potential $s$. We outline the differences between our expansion and the original one in the Appendix. Each diagram represents a certain convolution of fixed-end partition function factors $\tilde{Q}_{0}(\phi, s)$, and $\tilde{f}(p)$, the Fourier transform of $f(r)$. Each segment of the solid line in the diagrams corresponds to a $\tilde{Q}_{0}$ factor, and each dashed line to an $\tilde{f}$ factor. Thus, the diagram of Fig. $1(\mathrm{~g})$ corresponds to the expression

$$
\begin{aligned}
& \tilde{Q}_{0}(0, s) \int_{\left|\rho_{x}\right|,\left|p_{y}\right|, \ldots,<z / t} d^{d} l p /(2 \pi)^{d} \\
& \quad \times \tilde{f}(p) \tilde{Q}_{0}^{2}(p, s) \tilde{Q}_{0}(p+q, s) \tilde{Q}_{0}(0, s) .
\end{aligned}
$$

Figure 1 includes all topologically distinct diagrams with up to two dashed lines. The sum of the convolutions represented in Fig. 1 is equal to $\bar{Y}(q, s)$, expanded to second order in the Mayer factors $f$. We note the resemblance between these diagrams and, for example, those of Fig. III. 3 of Yamakawa's text. ${ }^{8}$ Our diagrams also resemble those of phase transition field theory. ${ }^{16,20}$ When expressed in the detailed form of Fig. 1, our diagrams require no symmetry factors; each diagram enters the sum with equal weight.

In the limit of large distances $q l \ll 1$ and long chains $s-s_{c} \ll 1$, the expansion may be simplified after restating it in terms of $s-s_{c}$ rather than $s$. Then $f(p)$ becomes independent of $p$ over the range of interest, and the boundaries of the loop integrations $\int d^{a} p$ recede to infinity. The change of variable from $s$ to $s-s_{c}$ causes a modification in the evaluation of the diagrams, as explained in the Appendix. In the long-chain limit the $Q_{0}(q, s)$ factors may be written in the simple form $\tau_{c} /\left(q^{2}+t\right)$, where the constant $\tau_{c} \equiv e^{s} c l^{-2}$, and $t \equiv 2 d l^{-2}$ $\times\left(s-s_{c}\right)$. With these changes, factors of $\tau_{c}$ and $l$ may be combined with the $\tilde{f}$ factors. Then the expansion for $\tilde{Y}$ becomes a factor $\tau_{c}^{3}$ times an expansion in $\tilde{f} \tau_{c}^{2} l^{d /(2 \pi)^{d}}$ $=\tilde{f} e^{2 s} c l^{d-4} /(2 \pi)^{4} \equiv u$. The diagrams are otherwise independent of $l$. Thus, to first order $\bar{Y}(q, t)$ takes the form

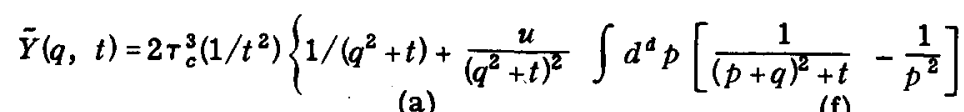

$$
\begin{aligned}
& \left.+\frac{2 u}{t\left(q^{2}+t\right)} \int d^{d} p\left(\frac{1}{p^{2}+t}-\frac{1}{p^{2}}\right)+\frac{2 u}{q^{2}+t} \int d^{d} p \frac{1}{\left(p^{2}+t\right)\left[(p+q)^{2}+t\right]}+u \int d^{d} p \frac{1}{\left(p^{2}+t\right)^{2}\left[(p+q)^{2}+t\right]}\right\},
\end{aligned}
$$

(b, c) 
where the letter in parentheses under each term indicates the diagram in Fig. 1 which corresponds to it. This may be condensed into the form

$$
\begin{aligned}
\tilde{Y}(q, t)= & 2 \tau_{c}^{3}\left(1 / t^{2}\right)\left[\frac{1}{q^{2}+t}-\frac{2 u I(t)}{q^{2}+t}-\frac{u t I(t)}{\left(q^{2}+t\right)^{2}}\right. \\
& \left.+\frac{2 u I(q, t)}{q^{2}+t}-\frac{u}{2} \frac{\partial I(q, t)}{\partial t}\right],
\end{aligned}
$$

where

$$
I(q, t) \equiv \int d^{d} p \frac{1}{\left(p^{2}+t\right)\left[(p+q)^{2}+t\right]}
$$

and $I(t) \equiv \int d^{d} p p^{-2}\left(p^{2}+t\right)^{-1}$.

\section{RENORMALIZATION}

This theory provides the continuum $\left(l \rightarrow 0 ; s \rightarrow s_{c}\right)$ behavior for weak repulsion, i.e., for small $u$. For the perturbation to be weak, the dimensionless interaction parameter $u t^{(d-4) / 2}$ must be small compared to 1 . At this stage the expansion is equivalent to that used by $\mathrm{Zimm}^{21}$ and others ${ }^{22,23}$ to calculate properties of polymers in nearly ideal solvents. For the excluded volume limit of fixed repulsion $u$ and long chains $(t \rightarrow 0)$ the expansion evidently cannot be used as it stands for $d=3$, since the interaction parameter diverges. However for $d \geq 4$ the parameter remains finite. To make the expansion tractable, we are led to consider $d \approx 4$.

The "renormalization" of the perturbation theory is a way of exploiting the $d \rightarrow 4$ limit of the expansion to obtain systematic approximations to the $d=3$ behavior. First we generalize the integrals $\int d^{d} p$ of the expansion to noninteger $d$, following a standard prescription. ${ }^{24}$ Thus, in terms of our $\epsilon \equiv 4-d$,

$$
\begin{aligned}
I(q, t)= & (1 / 2) s(d) \Gamma(2-\epsilon / 2) \Gamma(\epsilon / 2) \\
& \times \int_{0}^{1}\left[t+x(1-x) q^{2}\right]^{-\epsilon / 2} d x, \\
I(t)=(1 / 2) & s(d) \Gamma(2-\epsilon / 2) \Gamma(\epsilon / 2)(1-\epsilon / 2)^{-1} t^{-\epsilon / 2},
\end{aligned}
$$

where $\Gamma$ is Euler's gamma function and $s(d) \equiv\left[2 \pi^{d / 2} /\right.$ $\Gamma(d / 2)]$ is the surface area of a $d$-dimensional sphere. As $d \rightarrow 4$, the $t \rightarrow 0$ divergence becomes weaker, but the wave vector integrations become large $[\mathrm{Eq}$. (8)]. This latter divergence appears in $\mathrm{Eq}$. (9) as a pole in $\epsilon$ from $\Gamma(\epsilon / 2)$. These integration divergences hold the information about the anomalous scaling properties of the theory; to extract the information, one notes that the divergent parts may be absorbed into factors $z_{1}$ multiplying the chemical potential $t$, the interaction parameter $u$, and $\tilde{Y}$ itself. Thus, the expansion for $\tilde{Y}_{R} \equiv z_{1} \tilde{Y}$ is finite to all orders for $d \leq 4$ when expressed in terms of $t_{R} \equiv z_{2} t$ and

$$
u_{R} \equiv z_{3} u S(d) \text {. }
$$

The $z_{i}$ factors may be chosen independent of $t$ and $q$. One says that the perturbation theory is renormalizable. Thus, the integration divergences which appear as $d \rightarrow 4$ are handled in the same way as the $s_{c}$ divergences noted in the Appendix. As in that case, the divergences may be removed in each order by a redefinition of the variables. As with $s_{c}$, one may find which $z$ factors are

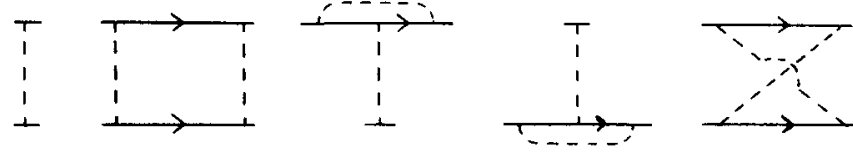

FIG. 2. Diagrams for the effective interaction $\Gamma^{(4)}$ to second order.

required by a detailed analysis of divergent subdiagrams. ${ }^{24}$

We first determine $u_{R}$. To lowest order in $u, z_{3}$ and thus $u_{R}$ [Eq. (10)] may be determined by requiring that the "effective interaction" $\Gamma^{(4)}$ be finite as $\epsilon-0$, when expressed in terms of $u_{R}$. The function $\Gamma^{(4)}\left(p_{1}, p_{2}, p_{3}\right.$, $\left.p_{4}\right)$ is defined as the sum of irreducible diagrammatic pieces to which four polymer lines of wavevector $p_{1}, \ldots$, $p_{4}$ may be attached (Fig. 2). Irreducible subdiagrams are those which cannot be separated into two parts by cutting a single polymer line. To next lowest order,

$$
\begin{aligned}
\Gamma^{(4)}\left(p_{1}, p_{2}, p_{3}, p_{4}\right)= & u\left\{1+u\left[I\left(p_{1}+p_{3}, t\right)+I\left(p_{3}+p_{4}, t\right)\right.\right. \\
& \left.\left.+I\left(p_{1}+p_{2}, t\right)+I\left(p_{1}+p_{4}, t\right)\right]\right\} .
\end{aligned}
$$

To make the integrals dimensionless, we express $p_{i}$ and $t$ as multiples of some inverse length parameter $\chi$ :

$$
p_{i}=\bar{p}_{i} \chi, \quad t=\bar{t} \chi^{2} .
$$

The parameter $\chi$ is chosen to be comparable to the wave vectors of interest in the problem; and thus in the excluded volume limit $\chi l \rightarrow 0$. Expanding $\Gamma^{(4)}$ near $d=4$ and inserting $u_{R}$, we obtain

$$
\begin{aligned}
\Gamma^{(4)} & =u_{R}\left[z_{3} s(d)\right]^{-1}\left\{1+\left(u_{R} \chi^{-\epsilon} / z_{3}\right)\left[\epsilon^{-1}(4-2 \epsilon)-(\epsilon / 2)\right]\right. \\
& \times \int_{0}^{1} d x\left[\ln \left(\bar{t}+x(1-x)\left(\bar{p}_{1}+\bar{p}_{3}\right)^{2}\right]+\ln \left[\bar{t}+x(1-x)\left(\bar{p}_{3}+\bar{p}_{4}\right)^{2}\right]\right. \\
& \left.+\ln \left[\bar{t}+x(1-x)\left(\bar{p}_{1}+\bar{p}_{2}\right)^{2}\right]+\ln \left[\bar{t}+x(1-x)\left(\bar{p}_{1}+\bar{p}_{4}\right)^{2}\right]+O\left(u_{R}^{2}\right)\right\}
\end{aligned}
$$

We now choose the first-order part of $z_{3}$ to cancel the $1 / \epsilon$ term: $z_{3}=1+(4 / \epsilon) g$ where we denote the dimensionless interaction $u_{R} \chi^{-\epsilon}$ as $g$.

These equations show that $g$ depends on $u$ and $\chi$ in the combination $u \chi^{-\epsilon} \sim \tilde{f}\left(l_{\chi}\right)^{-\xi}$, which goes to infinity, since $\chi l-0$ in the limit of interest.

To obtain $g$ in this limit, we cannot use Eq. (10) directly, but renormalization group theory ${ }^{24}$ shows that $(\partial g / \partial \ln \chi) \mid \gamma_{,}$, has a finite expansion in $g$. To second order one obtains

$$
(\partial g / \partial \ln \chi)=-g\left[\epsilon+(4) g+O\left(g^{2}\right)\right] .
$$

The expansion on the right side remains finite as $\chi \rightarrow 0$ and/or $\epsilon-0$. Indeed, this differential equation for $g(\chi)$ implies that as $\chi-0, g$ goes to a fixed finite value given by the vanishing of the [ ] expression above. Thus, $g \rightarrow-\epsilon / 4$. We denote the limiting value by $g^{*}$. Since the limiting value of the renormalized coupling $g *$ is of order $\epsilon$, one may consistently expand $\tilde{Y}_{R}$ or the exponents in $\epsilon$ using only a finite number of diagrams. 
One may then set $\epsilon=1(d=3)$ in the expansion to obtain useful estimates of $\tilde{Y}$. To order $\epsilon$ one finds, from Eq. (7)

$$
\begin{aligned}
\tilde{Y}_{R}\left(q, t_{R}\right)=2 \tau_{c}^{3} \chi^{-6} \bar{t}_{R}^{3}\left(1+\hat{q}^{2}\right)^{-1} \\
\quad \times\left(1+g\left\{\frac{\ln \bar{t}_{R}}{2\left(1+\hat{q}^{2}\right)}-1-\int_{0}^{1} d x \ln \left[1+x(1-x) \hat{q}^{2}\right]\right.\right. \\
\left.\left.\quad+\frac{1}{4}\left(1+\hat{q}^{2}\right) \int_{0}^{1} d x\left[1+x(1-x) \hat{q}^{2}\right]^{-1}+O(\epsilon)\right\}+O\left(g^{2}\right)\right),
\end{aligned}
$$

where $\bar{t}_{R} \equiv t_{R} \chi^{-2}$ and $\hat{q}^{2} \equiv q^{2} / t_{R}$, and the values $z_{1}^{-1 / 2}=z_{2}$ $=1-g /(\epsilon)$ of the renormalization factors have been used. This expression is consistent with the (independently known $)^{11,25}$ scaling law for $\tilde{Y}_{R}$, viz., $\bar{Y}_{R}$ $=t_{R}^{-\gamma-2} f_{2}\left(q t_{R}^{-\nu}\right)$, where $\gamma=1+\epsilon / 8+O\left(\epsilon^{2}\right)$ and $\nu=1 / 2+\epsilon / 16$ $+O\left(\epsilon^{2}\right)$. Comparing the perturbation expansion with the scaling law formula expanded in $q$ and $\epsilon$, one finds

$$
\begin{aligned}
{\left[f_{2}\left(q t^{-\nu}\right)\right]^{-1}=} & c_{1}\left\{1+[1+(\epsilon / 96)]\left(c_{2} q t^{-\nu}\right)^{2}\right. \\
& \left.+(\epsilon / 160)\left(c_{2} q t^{-\nu}\right)^{4}+O\left(\epsilon q^{8}\right)+O\left(\epsilon^{2}\right)\right\},
\end{aligned}
$$

where $c_{1}$ and $c_{2}$ are unknown (and nonuniversal) constant factors.

\section{RESULTS AND DISCUSSION}

The above prediction, describing as it does the correlations of a grand canonical chain-length distribution, cannot be compared directly with experiments. Thus, the formula above must be translated ${ }^{28}$ into the analogous formula for $\tilde{Y}$ for the experimental chain-length distribution. We first calculate the canonical $\vec{Y}_{n}(q)$ for chains of length $n$. The problem in general terms is to determine the coefficients $\alpha_{0}(n), \alpha_{2}(n)$, and $\alpha_{4}(n)$ in the limit of large $n$, given the coefficients $a_{0}, a_{2}$, and $a_{4}$ :

$$
\begin{aligned}
\tilde{Y}(q, t)= & a_{0} t^{-\gamma-2}+a_{2} t^{-\gamma-2-2 \nu} q^{2}+a_{4} t^{-\gamma-2-4 \nu} q^{4} \\
& +\cdots+a_{k} t^{-\gamma-2-k v} q^{k}+\cdots \\
\equiv & \sum_{n=0}^{\infty} e^{-s n} \tilde{Y}_{n}(q) \\
= & \sum_{n=0}^{\infty} e^{-s n}\left[\alpha_{0}(n)+\alpha_{2}(n) q^{2}+\alpha_{4}(n) q^{4}+\cdots+\alpha_{k}(n) q^{k}\right. \\
& +\cdots],
\end{aligned}
$$

where $t=c_{3}\left(s-s_{c}\right)$. This is readily accomplished, the nonintegral powers of $t$ giving rise to Euler $\Gamma$ functions involving the exponents:

$$
\alpha_{k}(n)=a_{k} c_{3}^{-\gamma-2-k \nu} n^{(\gamma+2)+k \nu-1} e^{s_{c} n} / \Gamma(\gamma+2+k \nu) .
$$

Since, for fixed $n, S_{n}(q)$ is just a fixed multiple of $\tilde{Y}_{n}(q)$, the ratio $\zeta$ defined after Eq. (2) is a ratio involving $\alpha_{0}$, $\alpha_{2}$, and $\alpha_{4}$ :

$$
\zeta=\left(\alpha_{4} / \alpha_{0}\right) /\left(\alpha_{2} / \alpha_{0}\right)^{2}=\kappa(\gamma, \nu)\left(a_{4} / a_{0}\right) /\left(a_{2} / a_{0}\right)^{2},
$$

where the constant $\kappa$ may be expressed in terms of $\kappa_{e}$ [Eq. (28)]:

$$
\begin{aligned}
\kappa(\gamma, \nu) & =[\Gamma(\gamma+2 \nu+2)]^{2} /[\Gamma(\gamma+4 \nu+2) \Gamma(\gamma+2)], \\
& =\frac{(\gamma+1+2 \nu)^{2}(\gamma+2 \nu)^{2} \kappa_{\varepsilon}(\gamma, \nu)}{(\gamma+1+4 \nu)(\gamma+4 \nu)(\gamma+1) \gamma} .
\end{aligned}
$$

TABLE I. The coefficients $\kappa_{e}[\mathrm{Eq},(28)]$ and $\kappa$ [Eq. (19)] computed using different approximations for $\gamma$ and $\nu$.

\begin{tabular}{lllll}
\hline \hline Approximation & $\nu$ & $\gamma$ & $\kappa_{\mathrm{B}}\left(\sim \zeta_{e}\right)$ & $\times(\sim \zeta)$ \\
\hline Ideal chain & $\frac{1}{2}$ & 1 & $\frac{1}{2}$ & $\frac{3}{4}$ \\
First order & $\frac{1}{2}(1+\epsilon / 8)$ & $1+\epsilon / 8$ & $\frac{1}{2}(1-\epsilon / 16)$ & $\frac{3}{4}(1-5 \epsilon / 96)$ \\
"Flory" & $\frac{3}{5}$ & 1 & 0.4072 & 0.6745 \\
"Best" & 0.588 & 1.1615 & 0.4544 & 0.694 \\
Swollen Gaussian & $\frac{3}{5}$ & 1 & $\frac{1}{2}$ & 0.829 \\
[see after Eq. (21)] & & & \\
\hline
\end{tabular}

The polydisperse ratio $\left(a_{4} / a_{0}\right)\left(a_{2} / a_{0}\right)^{-2}$ bears a simple relationship to the corresponding ratio for $1 / \bar{Y}$; indeed, if, for small $q,(\bar{Y})^{-1}=A_{0}+A_{2} q^{2}+A_{4} q^{4}+\cdots$, then $\left(a_{4} / a_{0}\right) /$ $\left(a_{2} / a_{0}\right)^{2}=1-\left(A_{4} / A_{0}\right) /\left(A_{2} / A_{0}\right)^{2}$. The right side is given by Eq. (15), giving

$$
\zeta=\kappa(\gamma, \nu)\left[1-\epsilon / 160+O\left(\epsilon^{2}\right)\right] \text {. }
$$

This result for $\zeta$ shows that one may obtain precise estimates for polymer correlation functions using renormalization methods. Using the best available estimates ${ }^{12}$ for $\gamma(1.162)$ and $\nu(0.588)$, one obtains $\zeta=0.690$ $\pm 1 \%$. Here the error estimate is based on the $O(\epsilon)$ correction in Eq. (21). If we set $d=4$, the self-repell ing chain behaves like the ideal chain: $\gamma \rightarrow 1, \nu \rightarrow 1 / 2$, and $\xi \rightarrow 3 / 4$. We recover the result of the swollen Gaussian method by neglecting the $O(\epsilon)$ correction in Eq. (21) and using Eq. (20) for $\kappa$, with $\gamma=1, \nu=3 / 5$, and $\kappa_{e}=1 / 2$, the ideal chain value. Table I illustrates that $\zeta$ is sensitive to the values of both $\gamma$ and $\nu$. To our knowledge this is the first prediction that relates the exponent $\gamma$ to $S(q)$. Figure 3 shows how this prediction appears in a plot of $S(q)$. To check the present predic tion clearly requires great experimental precision. From the graph one sees that over the range where the expansion of $S(q)$ to $O\left(q^{4}\right)$ is accurate, the ideal chain and the self-repelling chain differ only slightly from each other, when expressed in terms of the scaled variable $x$ [Eq. (2)]. The $S(q)$ of the self-repelling chain is smaller than for the ideal chain in this range. The reverse is true at large $q$, where the self-repelling $S(q)$ fall $s^{28}$ as $q^{-1 / \nu}$, while the ideal $S(q)$ falls faster, as $q^{-2}$. Thus, there must be some universal $x$ value at which the ideal and self-repelling $S(q)$ are equal.

Remarkably, our calculation shows that excludedvolume effects reduce $\zeta$. As mentioned above, this. contradicts the results of the swollen Gaussian chain hypothesis ${ }^{9,10}$ and Koyama's result. ${ }^{8}$ This difference can be understood ${ }^{27}$ as an effect of a "correlation hole" in the monomer-monomer distribution function. The existence of a correlation hole for the chain ends has first been pointed out by Des Cloizeaux. ${ }^{15}$ The same ef fect causes a reduction in $\xi$ for the end-end correlations as we show below.

The present method may be used to calculate arbitrary Taylor coefficients of $S(q)$. Each power of $q$ beyond $q^{2}$ has a universal coefficient analogous to $\zeta$. It is believed that the $\epsilon$ expansions for these are only asymptotic; but experience with phase transition problems indicates that the $O(\epsilon)$ and $O\left(\epsilon^{2}\right)$ estimates are reliable. To confirm the 


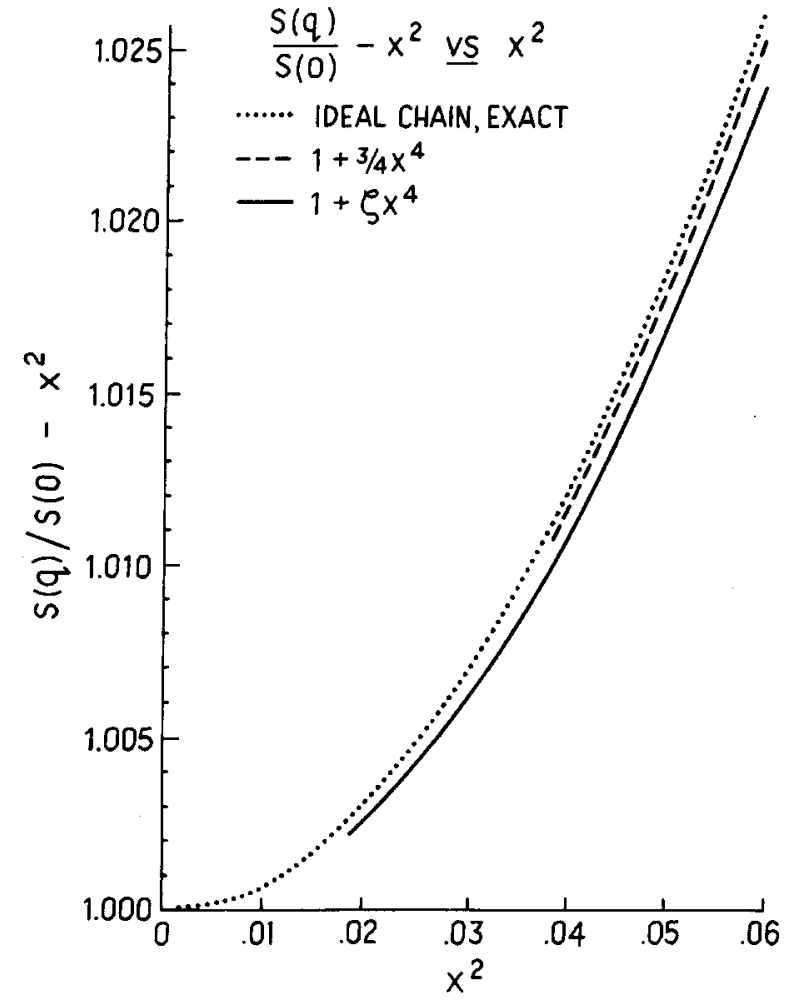

FIG. 3. Comparison of scaled $S(q)$ for ideal and self-repelling chains for small $q$. The variable $x^{2}=$ const. $q^{2}$ is defined to make $S(q)=S(0)\left[1+x^{2}+O\left(x^{4}\right)\right]$. The solid line is computed with $\zeta=0.690$, as calculated here.

precision of our result, we plan to extend our calculation to $O\left(\epsilon^{2}\right)$. Expansions to higher orders in $g *$ may be resummed using Borel summation methods to give very precise results. ${ }^{12,28}$

To calculate the value of $\zeta$ expected in a given experiment, one must know the fraction $P(n)$ of polymers in the sample of each length $n$. The quantity measured in a scattering experiment is the average $S(q)$ :

$$
\langle S(q)\rangle=\sum_{n} P(n) S_{n}(q)
$$

Since $S_{n}(q)$ has ${ }^{11}$ the form $n^{2} f\left(q n^{\nu}\right)$,

$$
\langle S(q)\rangle=\beta_{0} q^{0}\left\langle n^{2}\right\rangle+\beta_{2} q^{2}\left\langle n^{2+2 \nu}\right\rangle+\beta_{4} q^{4}\left\langle n^{2+4 \nu}\right\rangle+\cdots,
$$

where $\left(\beta_{4} / \beta_{0}\right)\left(\beta_{2} / \beta_{0}\right)^{-2}=\zeta$. The ratio $\langle\zeta\rangle$ of the measured $q$ coefficients is thus

$$
\langle\zeta\rangle=\zeta\left\langle n^{2+4 \nu}\right\rangle\left\langle n^{2}\right\rangle /\left\langle n^{2+2 \nu}\right\rangle^{2}
$$

The average $\langle\zeta\rangle_{I}$ for ideal chains is obtained by setting $\nu=1 / 2$.

Numerically, $\zeta$ proves somewhat sensitive to the chain length distribution. We have evaluated $\zeta$ for the Schulz ${ }^{29}$ distributions $-P(n) \sim n^{\sigma-1} e^{-c n}$, where $\langle\zeta\rangle$ may be expressed in terms of $\kappa(\gamma, \nu)$ [Eq. (19)]:

$$
\langle\zeta\rangle=\zeta / \kappa(\sigma, \nu) \text {. }
$$

Since the grand canonical $P(n)$ is itself a Schulz distribution, ${ }^{30}$ we may recover the grand canonical $\langle\zeta\rangle\left[=\left\langle a_{4}\right\rangle\right.$ $\left.\left.a_{0}\right)\left(a_{2} / a_{0}\right)^{-2}\right]$ by setting $\sigma=\gamma$ in Eq. (25). In Fig. 4 we plot $\langle\zeta\rangle$ for Schulz distributions vs the ratio $M_{w} / M_{n}$ of weight-averaged to number-averaged molecular weight. Evidently, the chain length distribution $P(n)$ must be well known in order to make a stringent test of our prediction. This sensitivity to $P(n)$ may be reduced by comparing $\langle\zeta\rangle$ with its ideal -chain value $\langle\zeta\rangle_{I}$. From the figure one sees that $\langle\xi\rangle-\langle\xi\rangle_{I}$ is relatively insensitive to $P(n)$. This difference may be readily measured by dissolving a single polymer sample in good and in theta solvents.

An alternate method for calculating $\zeta$ would be to $\epsilon$ expand $\bar{Y}_{n}(q)$ rather than $\bar{Y}(q, t)$. The recent formulation of Des Cloizeaux ${ }^{31}$ would allow this to be done directly. We may accomplish the same thing by $\epsilon$ expanding the factor $\kappa(\gamma, \nu)$ to first order. Then we obtain $\zeta=3 / 4(1-5 \epsilon / 96)$, some $2 \%$ greater than our best estimate: $\zeta=0.690$.

Our method may be readily adapted to calculating the end point structure function, the Fourier transform of the end point density $\sigma(x) .{ }^{11}$ The quantity $\zeta_{e}$ analogous to $\zeta$ may be found by first calculating the end-constrained partition function $\vec{Q}(q, t)$. For this quantity we may simply use Bray's ${ }^{32}$ extensive calculation developed for phase transitions. Bray's order parameter correlation function is proportional to $\tilde{Q}$ if the number of order parameter components is set to zero ${ }^{15}$ :

$$
\begin{aligned}
& \tilde{Q}\left(q, t_{R}\right)^{-1} \\
& \quad=c_{4} t_{r}^{-\gamma}\left\{1+\hat{q}^{2}-\eta \hat{q}^{4}\left[1.504+0.001+O\left(\epsilon^{2}\right)\right] / 100+O\left(\hat{q}^{6}\right)\right\},
\end{aligned}
$$

where $\eta=2-\gamma / \nu$ is of $O\left(\epsilon^{2}\right)$, and $\hat{q}$ is a constant times $q t^{-\nu}$. In three dimensions ${ }^{12} \eta=0.026 \pm 0.001$. Equation (26) is believed ${ }^{32}$ to be an excellent approximation for $d=3$. We analyze this function as we did $\tilde{Y}$ [Eq. (16)] to obtain

$$
\zeta_{e}=\kappa_{e}(\gamma, \nu)\left\{1+\eta\left[1.504+0.001 \epsilon+O\left(\epsilon^{2}\right)\right] / 100\right\},
$$

where

$$
\kappa_{e}(\gamma, \nu)=\Gamma^{2}(\gamma+2 \nu) /[\Gamma(\gamma+4 \nu) \Gamma(\gamma)] .
$$

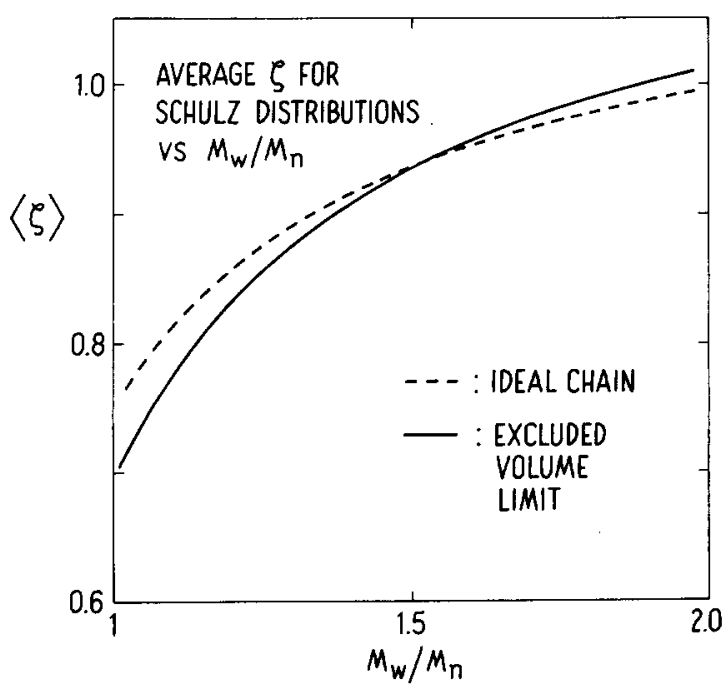

FIG. 4. The averages $\langle\zeta\rangle$ and $\langle\zeta\rangle_{I}$ for a Schulz distribution of excluded-volume or ideal chains plotted against the ratio of molecular weights $M_{w} / M_{n}$. 
This $\kappa_{e}$ differs from $\kappa$ because the formula for $\tilde{Q}$ analogous to Eq. (16) has an extra overall $t^{2}$ factor.

The end point ratio $\zeta_{e}$ is $1 / 2$ for a free chain. Using the best available exponents, one obtains 0.455 for the self-repelling chain. Expanding the exponents to first order in $\epsilon$, one obtains $\zeta_{e}=1 / 2-\epsilon / 32$, which is $3 \%$ smaller at $d=3$. Again the interaction reduces the ratio, in agreement with calculations for the nearly ideal chain. ${ }^{33}$ With a distribution $P(n)$ of chain lengths, the average $\zeta_{e}$ is given by

$$
\left\langle\zeta_{e}\right\rangle=\zeta_{e}\left\langle n^{4 \nu}\right\rangle\left\langle n^{2 \nu}\right\rangle^{-2}
$$

For a Schulz distribution of chain lengths of index $\sigma$ the moment ratio is $\left[\kappa_{e}(\sigma, \nu)\right]^{-1}$.

\section{ACKNOWLEDGMENTS}

We are grateful to Professor Robert Ullman and Jeffrey Prentis for several helpful suggestions on the manuscript.

\section{APPENDIX: GRAND CANONICAL AND CANONICAL FIXMAN EXPANSIONS COMPARED}

We illustrate the simplicity gained in the grand canonical formalism by computing $Y(y)$ for the ideal chain with both methods. In either formalism $Y(y)$ is the number of random chain configurations which pass through 0 and $y$. In both pictures $Y(y)$ is represented by the diagram in Fig. 1 (a) and is a product of ideal fixed-end chain partition functions $Q_{0}(x)$. In the canonical formalism the expression is

$$
Y_{n}(y)=2 \sum_{n_{1} n_{2}} \sum_{r_{0} \tau_{n}} Q_{0}\left(n_{1}, r_{0}\right) Q_{0}\left(n_{2}, y\right) Q_{0}\left(n-n_{1}-n_{2}, r_{n}-y\right) \text {. }
$$

In the grand canonical formalism the sums over $n_{1}$ and $n_{2}$ no longer appear:

$$
Y(y, s)=2 \sum_{r_{0} r_{n}} Q_{0}\left(r_{0}, s\right) Q_{0}(y, s) Q_{0}\left(r_{n}-y, s\right),
$$

where $Q_{0}(x, s) \equiv \sum_{n} e^{-s n} Q_{0}(x, n)$. By Fourier transforming $Y(y)$ to form $\tilde{Y}(q, s)$ one effects a further simplification:

$$
\tilde{Y}(q, s)=2 \tilde{Q}_{0}(0, s) \tilde{Q}_{0}(q, s) \tilde{Q}_{0}(0, s),
$$

where $\tilde{Q}_{0}(q, s) \equiv \sum_{x} e^{i \varepsilon^{*} x} Q_{0}(x, s)$. The same simplification seen here occurs throughout the grand canonical Fixman expansion. This is why we prefer the grand canonical method.

In the long chain limit $Q_{0}(x, n)$ has the form

$$
(2 d)^{n}\left[\pi /\left(4 n l^{2}\right)\right]^{4 / 2} e^{-x^{2} /\left(4 n l^{2}\right)} \text {. }
$$

This implies that for $q \ll l^{-1}$ and for $s$ near $\ln (2 d), \tilde{Q}_{0}(q$, $s)$ has the form

$$
2 d l^{-2} /\left[q^{2}+(s-\ln (2 d)) 2 d l^{-2}\right] \text {. }
$$

One may readily perform the Fixman expansion for long chains using the long chain form of $Q_{0}(x, n)$ shown above. However, one cannot directly perform the grand canonical expansion using the corresponding $\tilde{Q}_{0}(q, s)$. Certain integrations in the expansion diverge at large wave vec- tor. Thus, for example, in Fig. 1(e) the indicated integral $\int d^{d} p\left\{(p+q)^{2}+[s-\ln (2 d)] 2 d l^{-2}\right\}^{-1}$ diverges. The problem is that the chemical potential $s$ is not an appropriate variable. The divergences do not appear when the expansion is expressed in terms of $s-s_{c}(f)$, where $s_{c}(f)$ is the critical chemical potential, at which the partition function $\bar{Q}(q=0, s)$ becomes infinite. (At $s=s_{c}$ the average length $N$ must also become infinite.)

To see the effect of shifting $s_{c}$ on the diagrams we express the $\tilde{Q}_{0}$ factors in terms of their $s$-shifted counterparts:

$$
\tilde{Q}_{0}(q, s-\ln (2 d))=\bar{Q}_{0}\left(q, s-s_{c}\right)+\Delta \tilde{Q}_{0} .
$$

Here $\Delta \tilde{Q}_{0}$ is itself an expansion in powers of $f$ which may be found using the definitions of $\tilde{Q}_{0}$ and of $s_{c}$ above. Collecting like powers of $f$, including those from $\Delta \vec{Q}_{0}$, we have the desired modified expansion as a function of $s-s_{c}$. Then the terms may be arranged to show that the integrals are manifestly finite. Thus, for example, the divergent piece in Fig. 1 (f) noted above becomes the "subtracted" integral

$$
\int d^{d} p\left\{\left[(p+q)^{2}+\left(s-s_{c}\right) 2 d l^{-2}\right]^{-1}-p^{-2}\right\} .
$$

To calculate universal properties the actual value of $s_{c}$ is not needed. The process of shifting the $s$ variable, called "mass renormalization," is explained in Ref. 34.

${ }^{1}$ R. H. Ewart, C. P. Roe, P. Debye, and J. R. McCartney, J. Chem. Phys. 14, 687 (1946).

${ }^{2}$ J. P. Cotton, B. Farnoux, G. Jannink, J. Mons, and C. Picot, C. R. Acad. Sci. Ser. C 275, 175 (1972).

${ }^{3}$ P. Debye, J. Phys. Colloid, Chem. 51, 18 (1947).

${ }^{4}$ S. F. Edwards, Proc. Phys. Soc. London 85, 613 (1965).

${ }^{5}$ K. F. Freed, Adv. Chem. Phys. 22, 1 (1972).

${ }^{6} \mathrm{H}$. Yamakawa, Modern Theory of Polymer Solutions (Harper and Row, New York, 1971).

${ }^{7}$ D. S. McKenzie, Phys. Rep. C 27, 35 (1976); D. McIntyre, J. Mazur, and A. M. Wims, J. Chem. Phys. 49, 2887 (1968).

${ }^{8}$ R. Koyama, J. Phys. Soc. Jpn. 27, 1319 (1972).

${ }^{9}$ B. O. Ptitsyn, J. Phys. Chem. USSR 31, 1091 (1957).

${ }^{10} \mathrm{H}$. Benoit, C. R. Acad. Sci. 245, 2244 (1957).

${ }^{11}$ L. Schaefer and T. A. Witten, J. Chem. Phys. 66, 2121 (1977).

${ }^{12}$ J. C. Le Guillou and J. Zinn-Justin, Phys. Rev. Lett. 39, 95 (1977).

${ }^{13}$ J. P. Cotton, D. Decker, B. Farnoux, G. Jannink, R. Ober, and C. Picot, Phys. Rev. Lett. 32, 1179 (1974); Y. Miyaki, Y. Einaga, and H. Fujita, Macromolecules 11, 1180 (1978).

${ }^{14}$ P. G. De Gennes, Phys. Lett. A 38, 339 (1972).

${ }^{15} \mathrm{~J}$. Des Cloizeaux, Phys. Rev. A 10, 1665 (1974).

${ }^{16} \mathrm{~K}$. G. Wilson, and J. B. Kogut, Phys. Rep. C 12, 75 (1974).

${ }^{17}$ M. J. Fixman, J. Chem. Phys. 23, 1656 (1955).

${ }^{18}$ We use this lattice representation for the sake of concreteness and simplicity. Any description of the unperturbed chain which yields a Gaussian end-end distribution ${ }^{6}$ will serve as well. See L. Schaefer and T. A. Witten, J. Phys. (Paris) 41, $459(1980)$ for a description based on chains of Gaussian steps.

${ }^{19} \mathrm{~J}$. Mayer and M. G. Mayer, Statistical Mechanics (Wiley, New York, 1940).

${ }^{20} \mathrm{E}$. Brezin, J. C. Le Guillou, and J. Zinn-Justin, in Phase Transitions and Critical Phenomena, Vol. 6, edited by C. Domb and M. S. Green (Academic, New York, 1976).

${ }^{21}$ B. H. Zimm, J. Chem. Phys. 14, 164 (1946). 
${ }^{22}$ M. Fixman, Doctoral thesis, MIT, Cambridge, Mass. (1953).

${ }^{23}$ Y. Tagami and E. F. Casassa, J. Chem. Phys. 50, 2206 (1969).

${ }^{24} \mathrm{D}$. J. Amit, Field Theory, the Renormalization Group and Critical Phenomena (McGraw-Hill, New York, 1978).

${ }^{25}$ T. A. Witten and L. Schaefer, J. Phys. A 11, 1843 (1978).

${ }^{26}$ J. Des Cloizeaux, Phys. Rev, A 10, 1665 (1974).

${ }^{27}$ This explanation was suggested by Professor R. Uliman.

${ }^{28} \mathrm{C}$. Bervillier and C. Godreche, Phys. Rev, 21, 5427 (1980).
${ }^{29}$ G. V. Schulz, Z. Phys. Chem. Abt. B 43, 25 (1939).

${ }^{30}$ L. Schaefer, J. Phys. A 9, L71 (1976).

${ }^{31} \mathrm{~J}$. Des Cloizeaux, J. Phys. Lett. 41, L151 (1980).

${ }^{32}$ A. J. Bray, Phys, Rev. B 14, 1248 (1976).

${ }^{33} \mathrm{M}$. Kurata, H. Yamakawa, and E. Teramoto, J. Chem. Phys. 28, 785 (1958).

${ }^{34}$ R. D. Mattuck, A Guide in Feynmann Diagrams in the ManyBody Problem, 2nded. (McGraw-Hill, New York, 1976), Sec. 3, 3 . 